\title{
Deterministic optimization of the thermal Unit Commitment problem: A Branch and Cut search
}

\author{
Marian G. Marcovecchio ${ }^{\mathrm{a}, \mathrm{b}, *}$, Augusto Q. Novais ${ }^{\mathrm{b}}$, Ignacio E. Grossmann ${ }^{\mathrm{c}}$ \\ a INGAR/CONICET, Consejo Nacional de Investigaciones Científicas y Técnicas, Santa Fe, Argentina UNL, Universidad Nacional del Litoral, Santa Fe, Argentina \\ ${ }^{\mathrm{b}}$ LNEG, Laboratório Nacional de Energia e Geologia, Lisbon, Portugal \\ ${ }^{\mathrm{c}}$ Department of Chemical Engineering, Carnegie Mellon University, Pittsburgh, USA
}

\section{A R T I C L E I N F O}

\section{Article history:}

Received 31 July 2013

Received in revised form 6 March 2014

Accepted 17 March 2014

Available online 26 March 2014

\section{Keywords:}

Energy optimization

Unit Commitment problem

Deterministic optimization

Branch and Cut algorithm

\begin{abstract}
A B S T R A C T
This paper proposes a novel deterministic optimization approach for the Unit Commitment (UC) problem, involving thermal generating units. A mathematical programming model is first presented, which includes all the basic constraints and a set of binary variables for the on/off status of each generator at each time period, leading to a convex mixed-integer quadratic programming (MIQP) formulation. Then, an effective solution methodology based on valid integer cutting planes is proposed, and implemented through a Branch and Cut search for finding the global optimal solution. The application of the proposed approach is illustrated with several examples of different dimensions. Comparisons with other mathematical formulations are also presented.
\end{abstract}

(C) 2014 Elsevier Ltd. All rights reserved.

\section{Introduction}

The increasing electricity demand motivates the need to study different operational alternatives for planning power generation by integrating conventional generation sources with renewables, while ensuring profitability (Verhaegen, Meeus, Delvaux, \& Belmans, 2007; del Río, 2011; Ventosa, Baíllo, Ramos, \& Rivier, 2005; Xiao, Hodge, Pekny, \& Reklaitis, 2011); as well as, ways of improving the energy efficiency of existing power systems (Siirola \& Edgar, 2012). Furthermore, multi-period and multi-paradigm models have also been proposed in order to plan and optimize the energy system and components for a long time planning horizon (Hodge, Huang, Siirola, Pekny, \& Reklaitis, 2011; Zhang, Liu, Ma, Li, \& Ni, 2012; Zhang, Liu, Ma, \& Li, 2013). Recently, Soroush and Chmielewski (2013) have presented an overview of the state of the art and the current process systems opportunities in power generation, storage and distribution.

Planning the generation of electric power is based on three different classes of decisions defined according to the length of the planning time horizon: long-term decisions (capacity, type and number of power generators); medium term decisions (sched-

\footnotetext{
* Corresponding author at: INGAR/CONICET, Consejo Nacional de Investigaciones Científicas y Técnicas, Santa Fe, Argentina UNL, Universidad Nacional del Litoral, Santa Fe, Argentina. Tel.: +54 3424535568; fax: +54 3424553439.

E-mail address: mariangm@santafe-conicet.gov.ar (M.G. Marcovecchio).
}

uling of the existing units); short-term decisions (programming of the power that each committed unit must produce to meet the real-time electricity demand). These three levels of decision are usually referred to as Power Expansion, Unit Commitment (UC) and Economic Dispatch, respectively. The UC problem has been more widely studied due to its practical importance (Yamin, 2004; Padhy, 2004). Moreover, this problem has diverse applications in the chemical engineering area, for example in Mitra, Grossmann, Pinto, and Arora (2012) the UC constraints were applied to air separation plants to decide when to turn on and off compressors and liquefiers.

The UC can be formulated as a mathematical programming problem using different alternative models. Implementing schedulings based on the optimal solutions of these models, may result in significant economic savings. However, solving the UC problem is very difficult. In fact, this problem is a mixed integer programming problem, linear or nonlinear, that is well known to be NP-hard due to the exponential computational time that may be required in the worst case (Nemhauser \& Wolsey, 1988). A large effort has been spent over the last few decades to develop efficient methods capable of solving the UC problem for real industrial cases in practical computational times.

This paper focuses on the thermal UC problem. The solution methods proposed in the literature for solving this problem are either deterministic or heuristic. Approaches based on deterministic methods include: priority list (Senjyu, Shimabukuro, Uezato, \& Funabashi, 2003), integer mathematical programming (linear 